bound to recur unless the thrower changes his style completely, and uses extension of the elbow instead of medial rotation of the shoulder. These coaching points will be discussed in more detail, and with a great deal more authority, in the paper below by Colin Smith. (Fig. 4.)

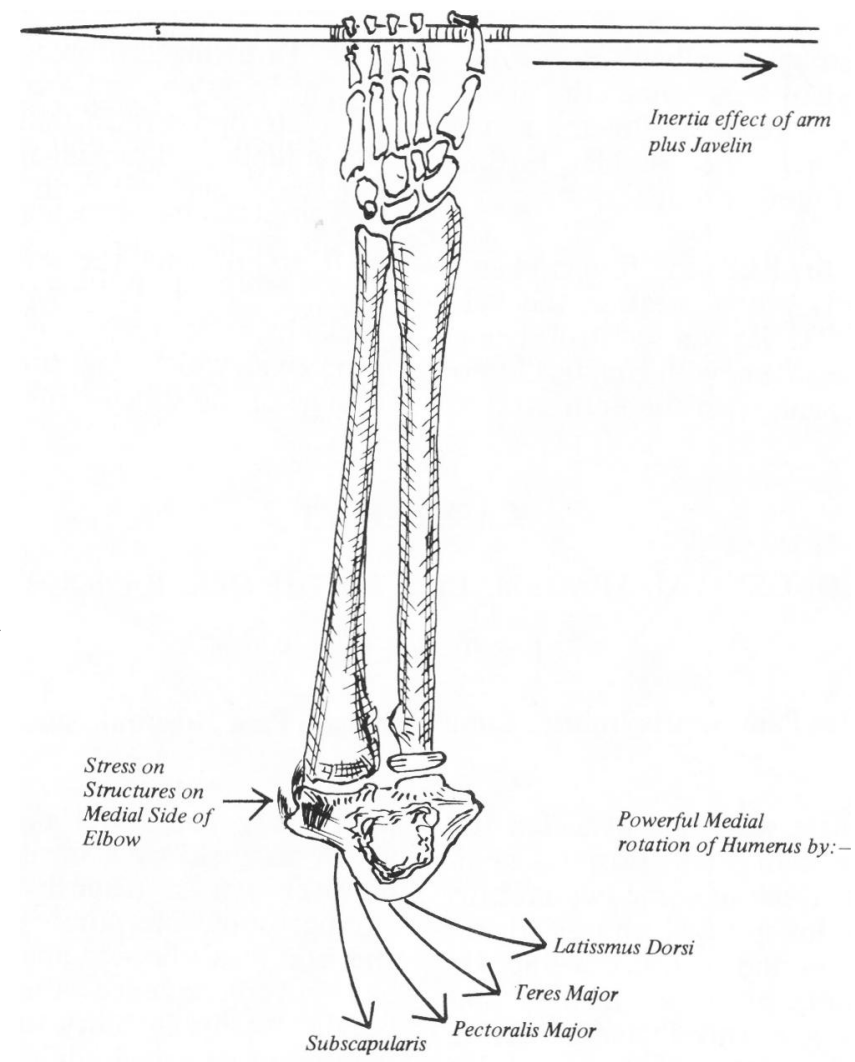

Fig. 4 Some Aetiological factors in "Javelin Elbow" when a round-arm action is used

BIOMECHANICS OF HITTING: BASIC USE OF THE ARM IN TENNIS

\title{
SUZAN LIVINGSTONE
}

\section{Beaconsfield School of Lawn Tennis}

The basic strokes used in the game of Lawn Tennis applied with or without a combination of spins are:-

1. Forehand Drive

2. Backhand Drive

3. Volleys (Forehand and Backhand)

4. Service

When coaching any of these basic strokes, one of the main difficulties encountered, as far as the arm is concerned, is teaching the player to have a complete racket control at all times during play.

Before this can be achieved, strong shoulder, upper arm and forearm muscles are necessary, as well as those of the wrist and hand. This is well shown by the majority of leading international players, particularly Rod Laver whose racket arm is very considerably more developed than the non playing arm, and when measured can be several inches larger in circumference.

During the time a player makes a stroke, it is desirable for the racket head to be kept higher than the level of the wrist. To do this when holding a racket weighing up to fourteen ounces, and when hitting a ball weighing approximately two ounces, considerable strength and control are required.

The three types of stroke consist of:- 
1. Swinging

2. Punching

3. Throwing

1. Swinging. Swinging 'through the ball' as in Forehand and Backhand groundstrokes, when the body should be turned sideways to the net, allowing a full swing forward, and swinging from the shoulder, hitting through the ball, with a long follow through.

2. Punching. Punching 'at the ball' as in forehand and backhand volleys, particularly when meeting the ball above the height of the net. Racket control being extremely difficult for the beginner, with practically no backswing or follow-through, and with the entire volley for the beginner played with a locked wrist. Grip strength required to 'stop' the racket immediately after impact is very considerable.

3. Throwing. Throwing the racket head through the ball, as in serving and smashing, the arm action being the complete opposite to that used for the volley. The serve is a complicated movement, with a long backswing, long follow-through and with maximum use of wrist action.

It is probably the serve which causes more injuries both to the arm (elbow joint, shoulder strain) and lower back. Incorrect technique is usually responsible for these problems, which can often be eased by a mechanical change of the actual stroke.

\title{
CASE REPORT
}

\section{A CONGENITAL ABNORMALITY OF THE OLECRANON FOSSA.}

\author{
J. G. P. WILLIAMS.
}

Farnham Park Sports Injuries Clinic, Wexham Park Hospital, Slough, Bucks.

S. L. is a 22 year old male who first attended the Sports Injury Clinic at Wexham Park Hospital complaining of pain in the right elbow of some two months duration. Pain was related to his sport which was cricket and in fact he felt it after a period of fast bowling. He stated that there was no history of specific incident and injury but that the pain had gradually increased during the preceding month. He also stated that one of his problems when fast bowling was that he tended to be called for throwing. The pain in the elbow was not related by him to any specific structure. He described it as a nagging sort of ache around the elbow joint itself. There was no history of swelling nor of any localised tenderness.

On examination the elbow looked normal. There was no obvious effusion nor any tenderness around the elbow joint, although there was perhaps a little slight tenderness over the tip of the olecranon. Flexion was full but extension was limited and there was a block of about 10 degrees. Pronation and supination at the elbow was full. There was no obvious cause for his trouble although with the history of a block to full extension it was thought that there might be a loose body present in the joint. The X-rays were taken which immediately demonstrated the cause of the trouble. S. L. had a presumably congenital anomaly in the olecranon fossa which was held by a small bone island. This bone island however was sufficiently large to interfere with the closing of the olecranon into its fossa in full extension and as a result he was unable to gain full extension. He had in consequence a mild traumatic synovitis and because of his inability fully to extend the arm he was tending to be called while fast bowling for throwing. In the case of this sort clearly any form of surgical intervention is unlikely to produce a more effectively functioning elbow than conservative management, particularly as the elbow caused no trouble apart from when he was bowling fast. He stated that he was not much good as a batsman and so we suggested that he might care to try spin bowling instead of fast bowling. He made the change with sufficient success for him to be able to continue playing cricket. He ceased to get any symptoms and he was no longer being called for no balling.

This case represents an interesting clinical oddity of no significance whatever in the context of normal day to day activities but which came for this patient to be a distinct drawback in the chosen area of his favourite sporting activity. 